PROCEEDINGS OF THE

AMERICAN MATHEMATICAL SOCIETY

Volume 127, Number 5, Pages 1443-1451

$\mathrm{S} 0002-9939(99) 04670-5$

Article electronically published on January 29, 1999

\title{
INTEGRABILITY OF SUPERHARMONIC FUNCTIONS, UNIFORM DOMAINS, AND HÖLDER DOMAINS
}

\author{
YASUHIRO GOTOH
}

(Communicated by Albert Baernstein II)

\begin{abstract}
Let $S^{+}(D)$ denote the space of all positive superharmonic functions on a domain $D \subset \mathbf{R}^{n}$. Lindqvist showed that $\log S^{+}(D)$ is a bounded subset of $B M O(D)$. Using this, we give a characterization of finitely connected

2-dimensional uniform domains and remarks on Hölder domains.
\end{abstract}

\section{Notation AND MAIN RESUlt}

Let $S^{+}(D)$ and $H^{+}(D)$ denote the spaces of all positive superharmonic and positive harmonic functions on a domain $D \subset \mathbf{R}^{n}, n \geq 2$, respectively. The quasihyperbolic metric $k_{D}$ on $D$ is defined by

$$
k_{D}(x, y)=\inf _{\gamma} \int_{\gamma} \frac{d s}{d(\cdot, \partial D)},
$$

where $d$ denotes the Euclidean distance, and the infimum is taken over all rectifiable curves $\gamma \subset D$ joining $x$ to $y$ (cf. [2]). We say that $D$ is a Hölder domain if

$$
k_{D}\left(x, x_{0}\right) \leq \frac{1}{\alpha} \log \left(2+\frac{1}{d(x, \partial D)}\right)+C, \quad x \in D,
$$

for some $\alpha, C>0$. Note that $D$ is a Hölder domain iff $\sup _{x \in D} \int_{B_{x}} e^{p k_{D}\left(\cdot, x_{0}\right)} d m<\infty$ for some $p>0$, where $B_{x}$ denotes the ball with center $x$ and radius $d(x, \partial D) / 2$, and $m$ denotes the $n$-dimensional Lebesgue measure. Smith-Stegenga showed the following remarkable characterization of Hölder domains, which asserts that the local exponential integrability of the quasihyperbolic metric implies the global one:

Proposition $1.1([9])$. If $D$ is a Hölder domain in $\mathbf{R}^{n}$, then $\int_{D} e^{p k_{D}\left(\cdot, x_{0}\right)} d m<\infty$ for some $p>0$.

Using this or a similar $B M O$ argument, Smith-Stegenga, Masumoto, and Stegenga-Ullrich investigated the $L^{p}$ integrability of $S^{+}(D)$ functions:

Proposition 1.2 ([10], [7], [11], cf. [12]). If $D$ is a Hölder domain in $\mathbf{R}^{n}$, then $S^{+}(D) \subset L^{p}(D)$ for some $p>0$. Conversely, if $D$ is a finitely connected subdomain of $\mathbf{R}^{2}$ and $S^{+}(D) \subset L^{p}(D)$ for some $p>0$, then $D$ is a Hölder domain.

Received by the editors May 7, 1997 and, in revised form, August 25, 1997.

1991 Mathematics Subject Classification. Primary 46E15.

Key words and phrases. BMO, quasihyperbolic metric, uniform domain, Hölder domain, superharmonic function, harmonic function.

(C)1999 American Mathematical Society 
Lindqvist clarified the argument of Stegenga-Ullrich by showing the following. Let $B M O(D)$ be the space of all locally integrable functions $f$ on $D$ satisfying

$$
\|f\|_{*, D}=\sup |B|^{-1} \int_{B}\left|f-f_{B}\right| d m<\infty,
$$

where the supremum is taken over all balls $B$ in $D,|B|=m(B)$, and $f_{B}$ denotes the integral mean of $f$ over $B$.

Proposition 1.3 ([4]). For an arbitrary subdomain $D$ of $\mathbf{R}^{n}$, we have

$$
\|\log u\|_{*, D} \leq C(n), \quad u \in S^{+}(D) .
$$

In our former paper [3], we obtained various estimations for the integrability of $B M O(D)$ functions. So, Lindqvist's theorem immediately provides the corresponding results for $S^{+}(D)$. Now we state one of them.

We say that a proper subdomain $D$ of $\mathbf{R}^{n}$ is a uniform domain if

$$
k_{D}(x, y) \leq C \log \left(2+\frac{d(x, \partial D)+d(y, \partial D)+|x-y|}{\min \{d(x, \partial D), d(y, \partial D)\}}\right), \quad x, y \in D,
$$

for some $C>0$. Each bounded uniform domain is Hölder. For $p>0$ and a measurable subset $E$ of $\mathbf{R}^{n}$, we set

$$
N_{p}(E)=|E|^{-1} \inf _{y \in \mathbf{R}^{n}}\left(\int_{E}|x-y|^{p} d m(x)\right)^{\frac{n}{n+p}} .
$$

$N_{p}(E)$ is invariant under similarities of $\mathbf{R}^{n}$, and a kind of distance between $E$ and balls. Then from [3], Theorem 5.3, we have

Theorem 1.1. If $D$ is a uniform domain in $\mathbf{R}^{n}$, then there exist constants $p_{0}, p$, $C>0$ such that for each $u \in S^{+}(D)$ and each measurable subset $E$ of $D$, we have

$$
\left(|E|^{-1} \int_{E} u^{p} d m\right)\left(|E|^{-1} \int_{E} u^{-p} d m\right) \leq C N_{p_{0}}(E)^{2} .
$$

Our main aim of the present paper is to show that the converse holds if $D$ is a finitely connected subdomain of $\mathbf{R}^{2}$ :

Theorem 1.2 (Main Theorem). Let $D$ be a finitely connected proper subdomain of $\mathbf{R}^{2}$. Assume that there exist constants $p_{0}, p, C>0$ such that for each $u \in S^{+}(D)$ and each measurable subset $E$ of $D$, we have

$$
\left(|E|^{-1} \int_{E} u^{p} d m\right)\left(|E|^{-1} \int_{E} u^{-p} d m\right) \leq C N_{p_{0}}(E)^{2} .
$$

Then $D$ is a uniform domain.

The proof of the Main Theorem is given in $\S 2$. In $\S 3$, we list some other immediate consequences of Lindqvist's theorem and of the author [3]. Using these results, we investigate the integrability of $S^{+}(D)$ functions on Hölder domains $(\S 4)$ and the boundedness of domains with some integrability condition for $S^{+}(D)(\S 5)$.

The author would like to thank the referee for his helpful comments and suggestions. 


\section{Proof of the Main Theorem}

To prove the Main Theorem, we need a lemma, which also plays a fundamental role in $\S \S 4$ and 5. From the Harnack inequality, $|\log u(x)-\log u(y)| \leq C k_{D}(x, y)$, $x, y \in D$, holds for each $u \in H^{+}(D)$, where $C=C(n)>0$. Conversely,

Lemma 2.1. Let $D$ be a finitely connected proper subdomain of $\mathbf{R}^{2}$. Assume that each boundary component contains more than two points. Then there exist constants $C_{1}, C_{2}>0$ such that for each pair of points $x_{1}, x_{2}$ on $D$, we can find a pair of an arc $\gamma$ joining $x_{1}$ to $x_{2}$ and $a H^{+}(D)$ function $u$ satisfying

$$
\int_{\gamma_{y z}} \frac{d s}{d(\cdot, \partial D)} \leq C_{1}(\log u(y)-\log u(z))+C_{2}, \quad y, z \in \gamma,
$$

where $\gamma_{y z}$ denotes the portion of $\gamma$ joining $y$ to $z$ and $y$ is between $z$ and $x_{2}$.

Proof. Since $k_{D}$ is conformally invariant modulo constant factors, we may assume that $D$ is a bounded domain surrounded by a finite number of circles, and that $x_{1}$ and $x_{2}$ are sufficiently close to some boundary components $F_{1}$ and $F_{2}$, respectively. We may assume $F_{1} \neq F_{2}$. A similar argument holds when $F_{1}=F_{2}$. We may also assume that $F_{1}=\{|x|=1\}$; the outer boundary of $D$, and $F_{2}=\{|x|=a\}$, $0<a<1$. Take $b>0$ so that $\{a<|x|<a+2 b\} \subset D,\{1-2 b<|x|<1\} \subset D$. Let $x_{j}=r_{j} e^{i \theta_{j}}, j=1,2$. Let $\gamma_{1}$ and $\gamma_{2}$ be the segments joining $x_{1}$ to $x_{1}^{\prime}=(1-b) e^{i \theta_{1}}$ and $x_{2}^{\prime}=(a+b) e^{i \theta_{2}}$ to $x_{2}$, respectively. We can take an $\operatorname{arc} \gamma^{\prime} \subset D$ joining $x_{1}^{\prime}$ to $x_{2}^{\prime}$ so that $\int_{\gamma^{\prime}} d(\cdot, \partial D)^{-1} d s \leq C$. Let $u$ be the Martin kernel function for $x_{2}^{\prime \prime}=a e^{i \theta_{2}} \in \partial D$, i.e.

$$
u(x)=\lim _{D \ni y \rightarrow x_{2}^{\prime \prime}}\left(g_{D}(x, y) / g_{D}\left(x_{0}, y\right)\right),
$$

where $g_{D}$ is the Green function on $D$ and $x_{0}$ is a fixed point on $D$. Then it is easy to check that $u(x) \approx(|x|-a)^{-1}, x \in \gamma_{2}, u(x) \approx 1-|x|, x \in \gamma_{1}$, and $u(x) \approx 1$, $x \in \gamma^{\prime}$. So $\gamma=\gamma_{1} \cup \gamma^{\prime} \cup \gamma_{2}$ and $u$ satisfy the required condition.

Proof of the Main Theorem. Assume that $D$ satisfies the condition of the Main Theorem. In general, if $D$ is uniform, then $D \backslash\{x\}, x \in D$, is also uniform. Thus we may assume that $D$ has no punctures. Let $x, y \in D$ and set $l=|x-y|$. We may assume that $d(x, \partial D) \leq d(y, \partial D) \leq l$. Let $r=d(x, \partial D) / 2$ and $B_{x}$ (resp. $\left.B_{y}\right)$ denote the ball with center $x(y)$ and radius $r$. Let $E=B_{x} \cup B_{y}$. From Lemma 2.1 , there exists a $H^{+}(D)$ function $u, u(y)=1$, satisfying

$$
k_{D}(x, y) \leq C_{1} \log u(x)+C .
$$

Then

$$
\int_{E} u^{p} d m \geq C r^{n} e^{p C_{1}^{-1} k_{D}(x, y)}, \quad \int_{E} u^{-p} d m \geq C r^{n},|E| N_{p_{0}}(E) \leq C\left(l^{p_{0}} r^{n}\right)^{\frac{n}{n+p_{0}}} .
$$

Hence

$$
e^{p C_{1}^{-1} k_{D}(x, y)} \leq C\left(\frac{l}{r}\right)^{\frac{2 n p_{0}}{n+p_{0}}},
$$

and so $D$ is a uniform domain. 


\section{SOME OTHER DIRECT CONSEQUENCES OF LINDQVIST'S THEOREM}

In the present section, we list some other consequences of Lindqvist's theorem and of the author [3]. For a weight $w$ on a ball $B$, we set

$$
M_{p}(w, B)= \begin{cases}\operatorname{ess}_{\sup _{B} w,} & p=\infty, \\ \left(|B|^{-1} \int_{B} w^{p} d m\right)^{\frac{1}{p}}, & p \neq 0, p \neq \pm \infty, \\ \exp \left(|B|^{-1} \int_{B} \log w d m\right), & p=0, \\ \operatorname{essinf}_{B} w, & p=-\infty\end{cases}
$$

$M_{p}(w, B)$ is a non-decreasing function of $p$. Let $\mathcal{A}(D)$ denote the space of all balls $B$ on $D$ satisfying $d(B, \partial D) \geq \operatorname{rad}(B)$, where $\operatorname{rad}(B)$ denotes the radius of $B$. We say that a weight $w$ on a domain $D$ satisfies the $A_{\infty}$ condition locally on $D$ $\left(w \in A_{\infty}^{\text {loc }}(D)\right)$ if $0<M_{1}(w, B) \leq K M_{0}(w, B)<\infty, B \in \mathcal{A}(D)$, for some $K>0$. The typical example of $A_{\infty}^{\text {loc }}(D)$ weights are given by

$$
w=d(\cdot, \partial D)^{\alpha}\left(k_{D}\left(\cdot, x_{0}\right)+1\right)^{\beta} u^{\gamma}, \quad \alpha, \beta \in \mathbf{R},-\infty<\gamma<\frac{n}{n-2}, u \in S^{+}(D) .
$$

Lemma 3.1. Let $u \in S^{+}(D),-\infty \leq p<\frac{n}{n-2}\left(\frac{n}{n-2}=\infty\right.$ if $\left.n=2\right)$, and $B \in \mathcal{A}(D)$. Then $0<M_{p}(u, B) \leq C M_{-\infty}(u, B)<\infty$, where $C=C(n, p)>0$.

Proof. Let $g_{D}(\cdot, y)$ be the Green function on $D$ with pole $y$. We may assume $1 \leq$ $p<\frac{n}{n-2}$. From the Harnack inequality, it is easy to check that $M_{p}\left(g_{D}(\cdot, y), B\right) \leq$ $C M_{-\infty}\left(g_{D}(\cdot, y), B\right), B \in \mathcal{A}(D)$. Since each $S^{+}(D)$ function can be approximated by an increasing sequence of Green potentials, we may assume that $u$ is a Green potential of a positive measure $\nu$ on $D$. Then

$M_{p}(u, B) \leq \int_{D} M_{p}\left(g_{D}(\cdot, y), B\right) d \nu(y) \leq C \int_{D} M_{-\infty}\left(g_{D}(\cdot, y), B\right) d \nu(y) \leq C M_{-\infty}(u, B)$.

If $w \in A_{\infty}^{\text {loc }}(D)$, then $\log w \in B M O(D)$. So Lemma 3.1 gives another proof of Lindqvist's theorem. Let $\phi$ be a non-negative, non-decreasing, continuous function on $[0, \infty)$ such that $\phi(t)>0, t>0$. We say that $\phi$ is tame if $\phi(t+1) \leq C \phi(t), t \geq 1$. Let $x_{0} \in D$, and let $B_{0}$ be the ball in $D$ with center $x_{0}$ and radius $d\left(x_{0}, \partial D\right) / 2$. Then from [3], Theorem 2.3, we have

Theorem 3.1. Let $\phi$ be tame, $D$ a proper subdomain of $\mathbf{R}^{n}, w$ a $A_{\infty}^{\text {loc }}(D)$ weight with constant factor $K$, and $E$ a measurable subset of $D$. Assume that

$$
\int_{E} \phi\left(p_{0} k_{D}\left(\cdot, x_{0}\right)\right) w d m<\infty
$$

for some $p_{0}>0$. Then for each $p, 0<p<C_{1} \min \left\{p_{0}, 1\right\}$, and each $u \in S^{+}(D)$, we have

$$
\int_{E} \phi\left(p\left|\log u-(\log u)_{B_{0}}\right|\right) w d m \leq C_{2}\left(\int_{B_{0}} w d m+\int_{E} \phi\left(p_{0} k_{D}\left(\cdot, x_{0}\right)\right) w d m\right),
$$

where $C_{1}=C_{1}(n, K, \phi)>0$ and $C_{2}=C_{2}\left(n, K, \phi, p_{0}\right)>0$. 
This result has an advantage in that it gives an estimation of $S^{+}(D)$ functions not only from above but also from below. It is to be noted that from Lemma 3.1, we may replace the constant $(\log u)_{B_{0}}\left(=\log M_{0}\left(u, B_{0}\right)\right)$ with $\log M_{p}\left(u, B_{0}\right),-\infty \leq$ $p<\frac{n}{n-2}$, in particular, with $\log \left(\min _{B_{0}} u\right)$ or $\log \left(u_{B_{0}}\right)$. As to $H^{+}(D)$ functions, Theorem 3.1 is rather trivial, because the pointwise version $\left|\log u(x)-\log u\left(x_{0}\right)\right| \leq$ $C k_{D}\left(x, x_{0}\right)$ holds by the Harnack inequality. In the case of $\phi(t)=e^{t}, t^{p}$, we have

Corollary 3.1. Let D, $w$ and $E$ be as above. Assume that $\int_{E} e^{p_{0} k_{D}\left(\cdot, x_{0}\right)} w d m<\infty$ for some $p_{0}>0$. Then for each $p, 0<p<C_{1} \min \left\{p_{0}, 1\right\}$, we have

$$
\int_{E} u^{ \pm p} w d m \leq C_{2}\left(u_{B_{0}}\right)^{ \pm p}\left(\int_{B_{0}} w d m+\int_{E} e^{p_{0} k_{D}\left(\cdot, x_{0}\right)} w d m\right), \quad u \in S^{+}(D),
$$

where $C_{1}=C_{1}(n, K)>0$ and $C_{2}=C_{2}\left(n, K, p_{0}\right)>0$.

Corollary 3.2. Let $D, w$ and $E$ be as above. Let $0<p<\infty$. Assume that $\int_{E} k_{D}^{p}\left(\cdot, x_{0}\right) w d m<\infty$. Then

$$
\int_{E}|\log u|^{p} w d m \leq C\left(\int_{B_{0}} w d m+\int_{E} k_{D}\left(\cdot, x_{0}\right)^{p} w d m\right), \quad u \in S^{+}(D), u_{B_{0}}=1,
$$

where $C=C(n, K, p)>0$.

As to the non-weighted case, from [3], Corollary 5.2, we have

Theorem 3.2. Let $D$ be a proper subdomain of $\mathbf{R}^{n}$ and $E$ a measurable subset of D. Assume that $\int_{E} e^{p_{0} k_{D}\left(\cdot, x_{0}\right)} d m<\infty$ for some $p_{0}>0$. Then $N_{p_{0}}(E)<\infty$ and for each $p, 0<p<C_{1} \min \left\{p_{0}, 1\right\}$, and each $u \in S^{+}(D)$, we have

$$
\left(\int_{E} u^{p} d m\right)\left(\int_{E} u^{-p} d m\right) \leq C_{2}\left(\left(|E| N_{p_{0}}(E)\right)^{2}+\left(\inf _{x_{0} \in D} \int_{E} e^{p_{0} k_{D}\left(\cdot, x_{0}\right)} d m\right)^{2}\right)
$$

where $C_{1}=C_{1}(n)>0$, and $C_{2}=C_{2}\left(n, p_{0}\right)>0$.

Note that, in general, we can omit neither the first term nor the second term on the right side of the inequality (cf. the Main Theorem).

\section{REMARKS ON THE HÖLDER DOMAIN}

In the present section, we give some characterizations of finitely connected Hölder domains in $\mathbf{R}^{2}$. First, we show the following analogy of Proposition 1.2. Recall that $B_{0}$ is the disk with center $x_{0}$ and radius $d\left(x_{0}, \partial D\right) / 2$.

Theorem 4.1. If $D$ is a Hölder domain in $\mathbf{R}^{n}$, then $\int_{D} u^{-p} d m \leq C\left(u_{B_{0}}\right)^{-p}$, $u \in S^{+}(D)$, for some $p, C>0$. Conversely, if $D$ is a finitely connected subdomain of $\mathbf{R}^{2}$ and $\int_{D} u^{-p} d m \leq C\left(u_{B_{0}}\right)^{-p}, u \in S^{+}(D)$, for some $p, C>0$, then $D$ is a Hölder domain.

Proof. Assume that $D \subset \mathbf{R}^{2}$ is finitely connected and that $\int_{D} u^{-p} d m \leq C\left(u_{B_{0}}\right)^{-p}$, $u \in S^{+}(D)$, for some $p, C>0$. Since positive constants are in $S^{+}(D), D$ must have finite area. In general, if $D$ is Hölder, then $D \backslash\{x\}, x \in D$, is also Hölder. So we may assume that $D$ has no punctures. Let $x \in D$. From Lemma 2.1, we can take $u \in H^{+}(D), u\left(x_{0}\right)=1$, so that $k_{D}\left(x, x_{0}\right) \leq-C_{1} \log u(x)+C$. Then

$$
d(x, \partial D)^{2} \exp \left(p C_{1}^{-1} k_{D}\left(x, x_{0}\right)\right) \leq C \int_{B_{0}} u^{-p} d m \leq C,
$$


so $D$ is Hölder. The remaining implication follows from Corollary 3.1 and Proposition 1.1 .

Next, we consider another class of harmonic functions. Let $Q L H(D)$ be the space of all harmonic, Lipschitz continuous functions $h$ on $D$ with respect to the quasihyperbolic metric endowed with the norm

$$
\|h\|_{L}=\sup _{x \in D}|\nabla h(x)| d(x, \partial D) .
$$

Then $Q L H(D)$ agrees with the space of all harmonic $B M O(D)$ functions, and the norms $\|\cdot\|_{*, D}$ and $\|\cdot\|_{L}$ are comparable with constant factors depending only on $n$. Smith-Stegenga [9] showed that a domain $D$ is a Hölder domain iff we can take $p>0$ so that $\int_{D} e^{p|f|} d m<\infty$ holds for each $f \in B M O(D),\|f\|_{*, D} \leq 1$. We show that we may replace $B M O(D)$ with its subspace $Q L H(D)$ under some additive condition:

Theorem 4.2. Let $D$ be a proper subdomain of $\mathbf{R}^{2}$ which is conformally equivalent to some Hölder domain. Assume that there exist constants $p, C>0$ such that

$$
\int_{D} e^{p\left|h-h\left(x_{0}\right)\right|} d m \leq C, \quad h \in Q L H(D),\|h\|_{L} \leq 1 .
$$

Then $D$ is a Hölder domain.

Lemma 4.1. Let $D$ be as above. Then there exist constants $C_{1}, C_{2}>0$ depending only on $D$ and $x_{0}$ such that for each $x \in D$ we can find a real $Q L H(D)$ function $h,\|h\|_{L} \leq 1, h\left(x_{0}\right)=0$, so that $k_{D}\left(x, x_{0}\right) \leq C_{1} h(x)+C_{2}$.

Proof. Since $k_{D}$ (and so $Q L H(D)$ ) is conformally invariant, we may assume that $D$ is a Hölder domain from the beginning. Let $x \in D$. Take $x^{\prime} \in \partial D$ so that $d(x, \partial D)=\left|x-x^{\prime}\right|$. Let $h(y)=\log \left|y-x^{\prime}\right|$. Then $\|h\|_{L} \leq C$ and

$$
\left|h(x)-h\left(x_{0}\right)\right| \geq \log \frac{1}{d(x, \partial D)}-C \geq C k_{D}\left(x, x_{0}\right)-C .
$$

Proof of Theorem 4.2. Assume that $D$ satisfies the condition of Theorem 4.2 with $p=p_{0}$. Let $x \in D$. Take a real $Q L H(D)$ function $h$ satisfying the condition of the lemma above. Let $B=\{y|| y-x \mid \leq d(x, \partial D) / 2\}$. Then $k_{D}\left(x, x_{0}\right) \leq C_{1} h(y)+C$, $y \in B$, and so for $p=p_{0} C_{1}^{-1}$, we have

$$
d(x, \partial D)^{2} e^{p k_{D}\left(x, x_{0}\right)} \leq C \int_{B} e^{p_{0} h} d m \leq C .
$$

Thus $D$ is a Hölder domain.

Combining Proposition 1.2, and Theorems 4.1, 4.2, we have

Corollary 4.1. For a finitely connected proper subdomain $D$ of $\mathbf{R}^{2}$, the following conditions are equivalent:

(1) $D$ is a Hölder domain;

(2) $\int_{D} u^{p} d m \leq C u\left(x_{0}\right)^{p}, u \in S^{+}(D)$, holds for some $p, C>0$;

(3) $\int_{D} u^{-p} d m \leq C u_{B_{0}}^{-p}, u \in S^{+}(D)$, holds for some $p, C>0$;

(4) $\int_{D} e^{p\left|h-h\left(x_{0}\right)\right| /\|h\|_{L}} d m \leq C, h \in Q L H(D)$, holds for some $p, C>0$. 
Note that since $\log \left|x-x_{1}\right|, x_{1} \in \partial D$, belongs to $Q L H(D)$, if $D$ satisfies the condition (4), then $D$ has finite area, and so is conformally equivalent to some Hölder domain.

Finally in the present section, we give a remark on Lipschitz domains. We say that a bounded domain $D$ in $\mathbf{R}^{n}$ is $k$-Lipschitz $(k>0)$ if $D$ and $\partial D$ are given locally by a Lipschitz function whose Lipschitz constant is at most $k$. Each Lipschitz domain is a uniform domain. For various estimations of $S^{+}(D)$ functions on Lipschitz domains, see Maeda-Suzuki [5], Masumoto [6], and Aikawa [1]. Let $\alpha=\alpha_{n}\left(\tan ^{-1}(1 / k)\right)$, where $\alpha_{n}$ denotes the maximal order of barriers (cf. [5]). Let $D$ be a $k$-Lipschitz domain. Then it is known that if

$$
0<p<\min \{n /(n+\alpha-2), 1 /(\alpha-1)\},
$$

then $\int_{D} u^{p} d m \leq C u\left(x_{0}\right)^{p}, u \in S^{+}(D)$, holds ([6], [1]). Moreover, from the estimation $u(x) \geq C d(x, \partial D)^{\alpha} u\left(x_{0}\right), u \in H^{+}(D)$, it is easy to see that if $0<p<1 / \alpha$, then $\int_{D} u^{-p} d m \leq C u\left(x_{0}\right)^{-p}, u \in H^{+}(D)$, holds.

\section{BOUNDEDNESS OF DOMAINS WITH SOME INTEGRABILITY CONDITION}

In the present section, we give another application of Lindqvist's theorem and [3]. We investigate integrability conditions for $H^{+}(D)$ which ensure the boundedness of $D$. Proposition 1.2 shows that if $D$ is a finitely connected plane domain, then the $L^{p}$ integrability of $H^{+}(D)$ is one such condition. Recall that $B_{0}$ is a ball with center $x_{0}$ and radius $d\left(x_{0}, \partial D\right) / 2$.

Theorem 5.1. Let $\phi$ be tame.

(1) Let $\int_{1}^{\infty} \phi(t)^{-1} d t<\infty$. Let $D$ be a finitely connected proper subdomain of $\mathbf{R}^{2}$ and at least one boundary component contain more than two points. Assume that

$$
\int_{D} \phi\left(p \log ^{+} u\right) d m \leq C_{1}, \quad u \in H^{+}(D), u\left(x_{0}\right)=1,
$$

holds for some $p, C_{1}>0$. Then for each $x \in D$ we can take an arc $\gamma$ on $D$ joining $x_{0}$ to $x$ so that $|\gamma| \leq C_{2}$, where $C_{2}=C_{2}\left(D, x_{0}, \phi, p\right)>0$. In particular, $D$ is bounded.

(1)' In (1), we may replace $\log ^{+} u$ with $\log ^{+} \frac{1}{u}$.

(2) Conversely, let $\int_{1}^{\infty} \phi(t)^{\frac{-1}{n-1}} d t=\infty$. Then there exists an unbounded proper subdomain D of $\mathbf{R}^{n}$ which is homeomorphic to an open ball satisfying

$$
\int_{D} \phi(p|\log u|) d m \leq C, \quad u \in S^{+}(D), u_{B_{0}}=1
$$

for some $p, C>0$.

Proof of Theorem 5.1. (2) follows from Theorem 3.1, Lindqvist's theorem, and [3], Theorem 6.1. Next, assume that $\phi$ and $D$ satisfy the condition in (1) with $p=p_{0}$. We may assume that $D$ has no punctures. Let $x \in D$. From Lemma 2.1, we can take a pair of an arc $\gamma: x=x(t), 0 \leq t \leq a$, joining $x_{0}$ to $x$ and a $H^{+}(D)$ function $u, u\left(x_{0}\right)=1$, satisfying $\int_{\gamma_{y}} \frac{d s}{d(\cdot, \partial D)} \leq C \log u(y)+C, y \in \gamma$. Let $t_{0}=0$ and set $t_{1}=\max \left\{t>t_{0}|| x(t)-x\left(t_{0}\right) \mid \leq d\left(x\left(t_{0}\right), \partial D\right) / 2\right\}$. If $t_{1}<a$, then set $t_{2}=\max \left\{t>t_{1}|| x(t)-x\left(t_{1}\right) \mid \leq d\left(x\left(t_{1}\right), \partial D\right) / 2\right\}$. Repeating this process, we 
obtain a sequence $0=t_{0}<t_{1}<\cdots<t_{k-1}<t_{k}=a$. We may assume $k \geq 4$. Set $x_{j}=x\left(t_{j}\right)$. Since $\int_{t_{j}}^{t_{j+1}} d(x(t), \partial D)^{-1}|d x(t)| \geq C, 0 \leq j \leq k-2$, we have

$$
j \leq C \int_{0}^{t_{j}} \frac{|d x(t)|}{d(x(t), \partial D)} \leq C_{1} \log u\left(x_{j}\right)+C, \quad 0 \leq j \leq k-1 .
$$

Let $B_{j}, j \geq 2$, be the ball with center $x_{j}$ and radius $r_{j}=d\left(x_{j}, \partial D\right) / 10$. Then $B_{j}$, $0 \leq j \leq k-1$, are disjoint. Thus, for $p=p_{0} C_{1}^{-1}$, we have

$$
\begin{aligned}
\sum_{j=2}^{k-1} r_{j} & \leq\left(\sum_{j=2}^{k-1} \phi(p j)^{-1}\right)^{1 / 2}\left(\sum_{j=2}^{k-1} \phi(p j) r_{j}^{2}\right)^{1 / 2} \\
& \leq C\left(\int_{p}^{\infty} \phi(t)^{-1} d t\right)^{1 / 2}\left(\int_{D} \phi\left(p_{0} \log ^{+} u\right) d m\right)^{1 / 2}
\end{aligned}
$$

Let $\gamma^{\prime}$ be the associated polygon joining $x_{0}, x_{1}, \ldots, x_{k}$. Then $\left|\gamma^{\prime}\right| \leq C \sum_{j=2}^{k-1} r_{j}$, hence (1) follows. Finally, if we take $u \in H^{+}(D), u\left(x_{0}\right)=1$, so that $\int_{\gamma_{y}} \frac{d s}{d(\cdot, \partial D)} \leq$ $-C \log u(y)+C, y \in \gamma$, and repeating the argument above, we get (1)'.

\section{Corollary 5.1.}

(1) Let $1<p<\infty$. Let $D$ be a finitely connected proper subdomain of $\mathbf{R}^{2}$ and at least one boundary component contain more than two points. Assume that

$$
\int_{D}\left(\log ^{+} u\right)^{p} d m \leq C, \quad u \in H^{+}(D), u_{B_{0}}=1,
$$

for some $C>0$. Then $D$ is bounded.

(1)' In (1), we may replace $\log ^{+} u$ with $\log ^{+} \frac{1}{u}$.

(2) Let $0<p \leq n-1$. Then, there exists an unbounded proper subdomain $D$ of $\mathbf{R}^{n}$ which is homeomorphic to an open ball satisfying

$$
\int_{D}|\log u|^{p} d m \leq C, \quad u \in S^{+}(D), u_{B_{0}}=1,
$$

for some $C>0$.

\section{REFERENCES}

1. H. Aikawa, Integrability of superharmonic functions and subharmonic functions, Proc. Amer. Math. Soc., 120 (1994), 109-117. MR 94b:31003

2. F. W. Gehring and B. G. Osgood, Uniform domains and the quasihyperbolic metric, J. Anal. Math., 36 (1979), 50-74. MR 81k:30023

3. Y. Gotoh, On global integrability of $B M O$ functions on general domains, to appear in J. Anal. Math.

4. P. Lindqvist, Global integrability and degenerate quasilinear elliptic equations, J. Anal. Math. 61 (1993), 283-292. MR 95b:35062

5. F. - Y. Maeda and N. Suzuki, The integrability of superharmonic functions on Lipschitz domains, Bull. London Math. Soc., 21 (1989), 270-278. MR 90b:31004

6. M. Masumoto, Integrability of superharmonic functions on plane domains, J. London Math. Soc., 45 (1992), 62-78. MR 93f:31002

7. M. Masumoto, Integrability of superharmonic functions on Hölder domains of the plane, Proc. Amer. Math. Soc., 117 (1993), 1083-1088. MR 93e:31001

8. H. M. Reimann and T. Rychener, Funktionen beschränkter mittelerer Oszillation, Lecture Notes in Math. 489, Springer, 1975. MR 58:23564

9. W. Smith and D. Stegenga, Exponential integrability of the quasihyperbolic metric on Hölder Domains, Ann. Acad. Sci. Fenn. Ser. A. I. Math. 16 (1991), 345-360. MR 93b:30016 
10. W. Smith and D. Stegenga, Sobolev imbedding and the integrability of harmonic functions on Hölder domains, (Proc. Interna. Conf. Potential Theory, Nagoya 1990), Walter de Gruyter, Berlin, 1992, pp. 303-313. MR 93d:46056

11. D. A. Stegenga and D. C. Ullrich, Superharmonic functions in Hölder domains, Rocky Mountain J. Math. 29 (1995), 1539-1556. MR 97j:31003

12. N. Suzuki, Note on the integrability of superharmonic functions, Proc. Amer. Math. Soc., 118 (1993), 415-417. MR 93g:31007

Department of Mathematics, National Defense Academy, Hashirimizu 1-10-20 YokoSUKA 239 , JAPAN

E-mail address: gotoh@cc.nda.ac.jp 